responses, and to clarify roles and responsibilities of the agencies involved.

Methods: To demonstrate that planning and training for a disaster such as a chemical, biological, or radiological incident with an all-agencies approach provides an effective, integrated response for major and serious releases of toxic substances. The provision of decontamination facilities outside of hospital emergency departments will assist in keeping staff, patients, and treatment facilities free from contamination.

Results: Outcomes in combating hazardous material incidents are improved when all agencies integrate and respond synchronously, especially when these agencies are used to working together on a daily basis. "Those who work together on a daily basis, tend to work well together in disasters"

Conclusion: An integrated, all-agency field response to hazardous material incidents in which each agency is familiar with the dangers of unprotected responses improves outcomes in the acute phase of disaster management.

Keywords: agencies; containment; decontamination; hazardous material incidents; hospital emergency department; integration; outcomes; planning; roles; responsibilities; training; training responder awareness Prehosp Disast Med 2002;17:s6-7.

\section{A Pragmatic Approach to Effective Pre-clinical Mass Decontamination \\ Chris Green}

Divisional Officer, Advisor to Her Majesty's Fire Services Inspectorate in CBR Procedures, United Kingdom

The proposed UK approach to the At Scene Mass Decontamination Procedures for Contaminated Casualties that have been developed since 11 September for Chemical, Radiological, and Biological incidents was described. The procedures to be adopted, methodology of decontamination, key areas of responsibility, and equipment requirements were outlined. The outlined procedure is not the current UK position, but builds upon the existing planning arrangements.

Events can be foreseen that traverse state boundaries, and therefore, it is recognized that the eventual methodology must satisfy international protocols. Therefore, the presenter encouraged searching questions and debate, to assist in testing the research that has been carried out, and to identify any areas of similarity or difference between national procedures.

Keywords: biological; chemical; contamination; decontamination; planning; protocols; radiological; terrorism; UK

Prehosp Disast Med 2002;17:s6.

\section{Preparedness for NBC Disaster in Norway Jan Karlsen \\ Consultant, Medical Department of Norwegian Civil Defense}

Through the experience of disasters, especially by Chernobyl nuclear plant incident in the USSR,
Scandinavia has developed its preparedness plan and equipped shelters at public institutions including hospitals. By the time of the terrorism attacks in New York and Washington, DC in September 2001, the Norwegian government and the Civil Defense prepared additionally for biological and chemical disasters with the provisions of personal protection equipment and development of a decontamination system.

Keywords: bioterrorism; chemical; decontamination; disasters; personal protection equipment; plan; preparedness; shelters; terrorism

Prebosp Disast Med 2002;17:s7.

\section{Planning to Reduce the Risk of Mass Casualties in Large-Crowd Events \\ Eric W. Williams \\ Medical Displan Victoria-Australia}

\section{Objectives:}

1. To outline the need for planning guidelines for event organizers and event approving authorities.

2. To address the health implications associated with the staging of mass-crowd events.

3. To ensure Public Health requirements for safe food, water and toileting are in place to reduce the risk of toxic contamination and infections.

4. To provide an effective medical response organization to cope with a disaster impact in large crowds.

Methods: Legislation for regulations and guidelines to assist event organizers and approving authorities are desirable to provide a safe and healthy event, and requires wide consultation and agreement with all emergency services and agencies to reduce the risk of casualties. Integrated, allagency, event planning that will ensure any shortfalls in the overall incident response plan are addressed.

Providing printed guidelines for approving authorities, event organizers, and the general public provides community awareness for an understanding of the issues involved.

On-site medical arrangements during events will allow for the rapid triage of casualties and first-aid treatment to be provided, thus reducing the necessity for casualty transfer to hospitals. Such arrangements will include a medical organization capable of providing mass casualty management to cope with a disaster impact in large crowds.

Results: Outcomes for staging a satisfactory event are improved greatly when an all-agency integrated planning process has been followed, and public health issues of safe food, water, toileting, and medical arrangements are adequately addressed.

Conclusion: An integrated, all-agency response to incidents included joint event planning has considered all of the on-site risk factors greatly improves the outcome for any required emergency response.

Keywords: event planning; food; guidelines; integration; mass gatherings; medical management; planning; public health implications; regulations; risk reduction; sanitation; triage; water

Prehosp Disast Med 2002;17:s7. 